\title{
Minireview
}

\section{Epigenetics as a mechanism driving polygenic clinical drug resistance}

\author{
RM Glasspool', JM Teodoridis' and R Brown*,I \\ 'Centre for Oncology and Applied Pharmacology, Glasgow University, CRUK Beatson Laboratories, Garscube Estate, Glasgow G6I IBD, UK
}

\begin{abstract}
Aberrant methylation of CpG islands located at or near gene promoters is associated with inactivation of gene expression during tumour development. It is increasingly recognised that such epimutations may occur at a much higher frequency than gene mutation and therefore have a greater impact on selection of subpopulations of cells during tumour progression or acquisition of resistance to anticancer drugs. Although laboratory-based models of acquired resistance to anticancer agents tend to focus on specific genes or biochemical pathways, such 'one gene: one outcome' models may be an oversimplification of acquired resistance to treatment of cancer patients. Instead, clinical drug resistance may be due to changes in expression of a large number of genes that have a cumulative impact on chemosensitivity. Aberrant CpG island methylation of multiple genes occurring in a nonrandom manner during tumour development and during the acquisition of drug resistance provides a mechanism whereby expression of multiple genes could be affected simultaneously resulting in polygenic clinical drug resistance. If simultaneous epigenetic regulation of multiple genes is indeed a major driving force behind acquired resistance of patients' tumour to anticancer agents, this has important implications for biomarker studies of clinical outcome following chemotherapy and for clinical approaches designed to circumvent or modulate drug resistance.
\end{abstract}

British Journal of Cancer (2006) 94, 1087-1092. doi:I0.1038/sj.bjc.6603024 www.bjcancer.com

Published online 2I February 2006

(c) 2006 Cancer Research UK

Keywords: chemotherapy; biomarkers; DNA methylation; drug resistance; epigenetics

With the increasing variety of options for the treatment of cancer, it is becoming essential that the choice of anticancer therapy, or optimal combination of therapies, is based not only on conventional clinical/pathological criteria but also on the molecular phenotype of the tumour. Many solid tumours are initially sensitive to chemotherapy, but the vast majority will recur or progress with ultimate failure of conventional cytotoxic chemotherapy treatment. In general, novel experimental therapies are first examined for efficacy in patients that have failed standard treatments and whose tumours have acquired resistance to cytotoxic drugs. The pattern of gene expression of a tumour that no longer responds to conventional treatment will be very different from that of the tumour at presentation due to selection of drugresistant subpopulations. However, we know very little about the molecular characteristics of tumours after conventional treatment failure or the underlying mechanisms that drive the acquisition of drug resistance (Agarwal and Kaye, 2003).

Laboratory-based studies have identified a wide variety of biochemical pathways and many hundred genes that can potentially influence response to treatment in tumour cells. Early work in drug resistance identified genes such as MDR1 (P-glycoprotein) (Gottesman, 1993) and p53 (Lowe et al, 1993) as crucial in determining drug resistance in experimental models of

*Correspondence: Professor R Brown; E-mail: r.brown@beatson.gla.ac.uk Received 8 November 2005; revised 31 January 2006; accepted I February 2006; published online 2I February 2006 in vitro cell lines or transgenic mice. However, there is relatively little evidence that, individually, these mechanisms are able to predict treatment outcome in a manner that is comparable to known prognostic markers such as stage, performance status and histological grade (Agarwal and Kaye, 2003; Hall et al, 2004). The variability in quality of prognostic and predictive biomarker studies can make reaching a consensus on the value of a given marker challenging and recent recommendations have emphasised the need for appropriate design and reporting of biomarker studies (http://www.cancerdiagnosis.nci.nih.gov/assessment/progress/ progress/remark.html). Furthermore, response to treatment is only one factor influencing clinical outcome, numerous other tumour characteristics, such as capacity for invasion/metastasis or escape from the immune response, will also have an impact and may do so irrespective of the therapies used, diluting any association between a marker of drug resistance and clinical outcome. For instance, in the case of ovarian cancer, one of the strongest prognostic markers associate with time to progression of a tumour after treatment is the number of infiltrating $\mathrm{T}$ cells (Zhang et al, 2003). However, while these factors may confound the analysis of drug-resistance mechanisms, it is also becoming apparent that 'one gene: one outcome' is an oversimplification for acquired resistance to treatment of cancer patients. Thus, it seems increasingly likely that clinical drug resistance is due to polygenic expression changes involving multiple mechanisms rather than to the alteration of a single pathway or gene.

An analogy can be made between clinical drug-resistance genes and cancer susceptibility genes. Cancer susceptibility genes such as 
retinoblastoma $(R B 1)$ and adenomatois polyposis coli $(A P C)$ were originally identified as rare, mutant alleles that significantly increase the risk of cancer when inherited through the germ line. More recently, it has been argued that the greater part of cancer predisposition may be due to a combination of weak genetic variants at many different loci rather than to single high penetrance genes (Balmain et al, 2003). Similarly, the combination of weak effects on drug resistance due to expression changes at many genes may be more significant than the effect of any single gene. Since most cytotoxic drugs have a low therapeutic index, additive effects of multiple low fold changes in drug resistance may be sufficient to cause clinical treatment failure. However, identification and evaluation of multiple, small additive effects on clinical outcome following chemotherapy will require robust and novel statistical and computational approaches that allow nonrandom clustering of effects to be identified. In order to avoid the pitfalls inherent in analysing high-dimensional data sets such as multiple testing and limited sample size, large-scale prospective clinical studies are required. In addition, it may be more informative to study tumours longitudinally, as they acquire resistance during treatment rather than simply sampling tumours at presentation and to use surrogate end points more specific to drug resistance, such as response rather than overall survival.

\section{GENETIC VS EPIGENETIC ALTERATIONS OF RESISTANCE GENES}

At the time of writing, we have been unable to identify any study of clinical material that has identified acquisition of a $p 53$ mutation during treatment of a given patient and similarly gene amplification of $M D R 1$, although widely observed in highly resistant cell lines, is only rarely observed following chemotherapy. Therefore, although mutations in genes such as MDR1 and $p 53$ confer drug resistance in vitro and in animal models, and they may have a role in inherent resistance, there is little evidence that such genetic changes have a role in acquired clinical resistance following anticancer therapy.
It is clear that changes in gene expression do occur following chemotherapy leading to the question, if not gene mutations, what are the mechanisms leading to changes in gene expression? The answer may lie in the increasing evidence that epigenetic changes can be a crucial driving force behind the acquisition of drug resistance (Teodoridis et al, 2004). Indeed studies of drug-resistant cell line models have shown that multiple changes in methylation of $\mathrm{CpG}$ islands and epigenetic regulation occur following drug selection (Wei et al, 2003).

Epigenetics changes are heritable changes in gene expression that do not involve an alteration in the DNA sequence. Within the nucleus, DNA is packaged, together with histone proteins, into a higher order structure known as chromatin. Interpretation of genetic information coded within the DNA is regulated by mechanisms that involve stable and heritable modifications of DNA and histones. These modifications include methylation of DNA at CpG dinucleotides and methylation, acetylation and phosphorylation of histones. Changes in the patterns of these modifications are associated with chromatin remodelling and can result in changes in gene expression through increasingly understood mechanisms (Lachner et al, 2003).

DNA methylation involves the transfer of a methyl group to the carbon-5 position of cytosine residues, and occurs almost exclusively at cytosines that are followed by a guanine (CpG dinucleotides). CpG dinucleotides are relatively rare in the bulk of the genome and are nearly always methylated, but small stretches of DNA occur that are rich in CpG dinucleotides, so called CpG islands. These are usually unmethylated in normal cells and are often associated with the promoter regions of genes (Hendrich and Bird, 2000). Methylation of cytosines within these islands is associated with binding of methyl-binding domain (MBD) proteins, recruitment of histone deacetylases (HDACs) and histone methyltransferases, histone modification, chromatin condensation and transcriptional inactivation of the associated genes. A large number of genes where aberrant methylation of $\mathrm{CpG}$ islands within their promoters is associated with gene inactivation have now been identified in tumours (for methods of analysing CpG island methylation, see Box 1). These include genes involved in all aspects of tumour development and also in response to treatment

\footnotetext{
Box I Methods for detecting CpG island methylation

Methods for the analysis of CpG-island methylation are available both genome-wide and at the single gene level. Restriction landmark genomic scanning (RLGS) is performed by digesting genomic DNA with a methylation-sensitive restriction enzyme, end labelling of the resulting DNA fragments and subsequent digestion with two different restriction enzymes and 2-dimensional gel electrophoresis (Costello et al, 2000). Comparison of signal intensities between tumour and normal DNA after autoradiography allows estimation of the number of aberrantly methylated CpG islands in tumours, and individual aberrantly methylated CpG islands can be identified by sequencing. Differential methylation hybridisation $(\mathrm{DMH})$ is an alternative means of examining genome-wide methylation patterns that uses restriction digestion of genomic DNA and ligation to linkers (Huang et al, 1999), followed by digestion with a methylation-sensitive restriction enzyme such as BstUI, PCR amplification and hybridisation to CpG-rich DNA sequences (representing putative CpG islands). Comparison to hybridisation signals obtained from undigested linker-ligated DNA allowed the identification of aberrantly methylated CpG islands. Methylation sensitive-representational difference analysis (MS-RDA) uses genomic tester and driver DNA samples digested with the methylation-sensitive restriction enzyme Hpall (Ushijima et al, 1997). Sequences that are specific for the tester amplicon are subsequently enriched by repeated cycles of subtractive hybridisations.

Several methods for the analysis of the methylation status of individual CpG islands utilise bisulphate treatment of DNA, which has been described in detail (Grunau et al, 200 I; Warnecke et al, 2002). Bisulphite treatment of DNA converts unmethylated cytosines into uracil but does not affect methylated cytosines. A difference in methylation is thus converted into a difference in sequence. A widely used method for analysing the methylation status of specific sequences is methylation-specific PCR (MSP) (Herman et al, 1996). Methylation-specific PCR is performed using primers specific for either unmethylated or methylated sequences, thereby allowing the detection of the respective methylation state. Among the advantages of MSP are its easy detection due to its gain-of-signal character and its high sensitivity, allowing the detection of as little as $0.1 \%$ methylation in a DNA sample (Herman et al, 1996). The MethyLight technique also involves bisulphite modification. Fluorescence-based PCR is then performed with primers that either overlap CpG methylation sites or that do not overlap any CpG dinucleotides. Sequence discrimination can occur either at the level of the PCR amplification process or at the level of the probe hybridisation process or both (Eads et al, 2000). Combined restriction analysis (COBRA) uses primers that amplify the template irrespective of its methylation state (Xiong and Laird, 1997). The PCR product should therefore be heterogeneous and reflect the various methylation states present in the template. Discrimination of methylation states is achieved by restriction digest using a restriction site whose presence after bisulphite modification depends on the methylation state of the DNA. Combined restriction analysis allows the quantification of the methylation, but its disadvantage is that the methylation of one CpG site is not necessarily representative for the other CpG sites in the analysed sequence. The highest accuracy of methylation density in a region of DNA is achieved by bisulphite sequencing. As in COBRA, the modified DNA is amplified irrespective of its methylation state, but subsequently the amplicon is subcloned and sequenced. This not only allows detection of methylation with a single-nucleotide resolution but also gives information about the distribution of methylated cytosines within individual DNA molecules. The disadvantage is that bisulphite sequencing is relatively labour-intensive.
} 
(Teodoridis et al, 2004). Furthermore, for many genes such as $h M L H 1, B R C A 1$ and E-CADHERIN, aberrant methylation of CpG islands is a far more frequent mechanism of gene inactivation in sporadic tumours than gene mutation or deletion.

Gene inactivation by DNA methylation can occur at a rate several orders of magnitude higher than inactivation of the same gene by mutation (Bhattacharyya et al, 1994). So, if inactivation of a gene is an important mechanism driving the acquisition of drug resistance, the probability of this occurring by methylation and being selected for during chemotherapy is much more likely than it occurring by mutation. It has also been suggested that some tumours may acquire a CpG island methylator phenotype, that is, concurrent methylation of genes occurring in a nonrandom manner (Toyota et al, 1999). Cellular acquisition of a methylator phenotype could give cells a higher probability of cell transformation during carcinogenesis, as has been proposed for gene mutations and the mutator phenotype (Loeb, 1994). Disruption of the cellular processes involved in methylation could lead to concurrent hypermethylation of multiple genes, including tumour suppressor genes, and as a result lead to oncogenic transformation. A possible consequence of this would be that in a tumour with a methylator phenotype there would also be a higher probability of multiple drug-resistance/sensitivity genes becoming methylated, with associated changes in gene expression. Thus, epigenetic silencing may occur fortuitously during tumour development and only confer an advantage to tumour cells when they are treated with chemotherapy or radiotherapy. However, the existence of a distinct methylator phenotype has been challenged, since a bimodal distribution of methylation frequency has not been seen in the same way as observed for gene mutation in tumour cells with the mutator phenotype (Yamashita et al, 2003; Anacleto et al, 2005).

Nevertheless, the vast majority of tumours, if not all, have aberrant DNA methylation at $\mathrm{CpG}$ islands and epigenetic silencing of the associated genes. Patterns of $\mathrm{CpG}$ island methylation differ between and within tumour types in a manner that suggests that methylation is not a random process (Costello et al, 2000; Esteller et al, 2001; Wei et al, 2002). Epigenetic inheritance of transcription patterns has been implicated in the control of cell proliferation during development, as well as in stem-cell renewal and cancer (Valk-Lingbeek et al, 2004). However, the mechanisms and selective processes that give rise to specific methylation patterns in tumours remain unclear and are likely to be complex. Changes in cell metabolism (Paz et al, 2002), 'epigenetic drift'(Egger et al, 2004) and ageing (Richardson, 2002) have all been proposed. For instance, there is a global decrease in global 5 methlycytosine levels in DNA as cells age which is similar to that observed in many tumours (Richardson, 2002). At the same time, localised hypermethylation occurs at some $\mathrm{CpG}$ islands. In a restriction landmark genome scanning study of $\mathrm{CpG}$ island methylation in $\mathrm{T}$ lymphocytes from newborn, middle age and elderly subjects, only 29 of more than 2000 loci examined were found to alter methylation with ageing, with 23 increasing methylation, and six decreasing. The same subset also changed methylation status with age in the oesophagus, lung and pancreas, but in variable directions (Tra et al, 2002). Thus, age-specific methylation also occurs in a nonrandom manner suggesting a tightly controlled process. What ever the process, it seems likely that epigenetic changes regulating gene expression offer a more rapid means by which tumour cells can adapt to new environment such as cytotoxic drug therapy than genetic change and because such changes are heritable they can be passed on to daughter cells without the need for continuous selection pressure producing persistent acquired resistance.

\section{EVIDENCE FOR THE ROLE OF EPIGENETIC MECHANISMS IN DRUG RESISTANCE}

Altered expression of genes involved in apoptosis and DNA repair may play an important role in determining response to treatment and there are many examples of such genes being methylated in tumours (see Table 1). However, methylation of individual genes may have opposing effects on drug sensitivity. For instance, methylation of DNA repair genes such as MGMT and FANCF may lead to inactivation of DNA repair and confer chemosensitivity, while methylation and epigenetic silencing of proapoptotic genes such as $h M L H 1$ and APAF1 would confer resistance (Esteller et al, 2000; Soengas et al, 2001; Taniguchi et al, 2003; Teodoridis et al, 2004).

Table I Examples of genes associated with drug resistance

\begin{tabular}{|c|c|c|c|}
\hline Gene & Function & Evidence for role in drug sensitivity & Reference \\
\hline Apaf I & $\begin{array}{l}\text { Proapoptotic, binds and promotes } \\
\text { caspase } 9 \text { activation }\end{array}$ & $\begin{array}{l}\text { Methylation in melanoma cells can be reversed by DNMT inhibitors and this is } \\
\text { associated with increased sensitivity to doxorubicin }\end{array}$ & Soengas et al (200I) \\
\hline Caspase 8 & Proapoptotic & $\begin{array}{l}\text { Frequently methylated in tumours. Reversal of methylation associated with } \\
\text { increased sensitivity to doxorubicin, etoposide and cisplatin in Ewings sarcoma, } \\
\text { neuroblastoma, medulloblastoma and melanoma cell lines }\end{array}$ & Fulda et al (200I) \\
\hline hMLHI & DNA mismatch repair protein & $\begin{array}{l}\text { Methylation and loss of expression associated with resistance to cisplatin in cell } \\
\text { lines, which can be reversed by demethylation with decitabine. Increased } \\
\text { frequency of methylation after chemotherapy. Acquisition of } h \mathrm{MLHI} \\
\text { methylation during chemotherapy is independently associated with poor overall } \\
\text { survival in ovarian patients }\end{array}$ & Gifford et al (2004) \\
\hline FancF & $\begin{array}{l}\text { Activates DNA repair complex } \\
\text { containing BRCAI, and BRCA2 loss } \\
\text { cause a decreased ability to repair } \\
\text { chemotherapy-induced damage }\end{array}$ & $\begin{array}{l}\text { Methylation observed in cells with a defective BRCA2 pathway and increased } \\
\text { sensitivity to cisplatin. Demethylation of FANCF with decitabine reduced } \\
\text { sensitivity towards cisplatin in these cell line models }\end{array}$ & Taniguchi et al (2003) \\
\hline MGMT & $\begin{array}{l}\text { Removes mutagenic alkyl-groups } \\
\text { from the O6-position of guanine }\end{array}$ & $\begin{array}{l}\text { Methylation and associated loss of expression correlates with response to } \\
\text { temozolamide and BCNU in primary gliomas and overall and progression-free } \\
\text { survival in patients with diffuse large B-cell lymphoma treated with } \\
\text { cyclophosphamide-containing regimens }\end{array}$ & Paz et al (2004) \\
\hline $\mathrm{MCJ}$ & Unknown & $\begin{array}{l}\text { Methylation associated with poor response to therapy and poor overall survival } \\
\text { in ovarian patients }\end{array}$ & Strathdee et al (2005) \\
\hline $\mathrm{ER} \beta$ & & $\begin{array}{l}\text { Methylated in } 50 \% \text { of invasive breast cancers. Methylation of ER } \beta \text { less frequent } \\
\text { and expression rate was higher in tamoxifen-resistant compared to control } \\
\text { tumours }\end{array}$ & Chang et al (2005) \\
\hline
\end{tabular}


The DNA mismatch repair protein, hMLH1, has been shown to be necessary for engagement of a variety of downstream cellular responses to alkylating agents and cisplatin-induced DNA damage (Papouli et al, 2004). Re-expression of hMLH1 in isogenic model systems has demonstrated that loss of hMLH1 expression confers resistance to alkylating agents and cisplatin. The frequency of $h M L H 1$ methylation in ovarian tumours increases after chemotherapy (Strathdee et al, 1999). Tumours frequently release DNA which can subsequently be isolated from plasma samples (Johnson and Lo, 2002). Genetic and epigenetic changes that are present in the tumour can be detected in tumour DNA isolated from plasma. Analysis of $h M L H 1$ methylation in tumour DNA isolated from plasma of patients with ovarian cancer before chemotherapy and at relapse showed that $25 \%$ of patients acquired $h M L H 1$ methylation during chemotherapy and acquisition of $h M L H 1$ methylation was independently associated with poor overall survival, potentially as a result of poor response to subsequent lines of chemotherapy (Gifford et al, 2004).

In contrast to proapoptotic genes, loss of expression of DNA repair genes may be associated with increased sensitivity to chemotherapy. The DNA repair enzyme MGMT (O6 methyl guanine methyltransferase) removes mutagenic alkyl-groups from the O6-position of guanine, which could otherwise lead to $G \rightarrow A$ transitions after DNA replication (Gerson, 2004). As a result, it inhibits the killing of tumour cells by alkylating agents. Hypermethylation of the MGMT promoter and associated loss of expression correlates with response to temozolamide and BCNU in primary gliomas (Esteller et al, 2000; Paz et al, 2004) and is an independent predictor of overall and progression-free survival in patients with diffuse large B-cell lymphoma treated with cyclophosphamide-containing regimens (Esteller et al, 2002). Importantly, the methylation status of MGMT in gliomas at presentation does not correlate with the clinical response when temozolamide is used at relapse, demonstrating that the value of biomarkers may depend on when during tumour progression or treatment they are measured.

There is thus growing evidence that $\mathrm{CpG}$ island methylation of genes with a known direct involvement in drug responses has a potential role in predicting clinical outcome following chemotherapy. However, there is a need for studies to investigate the potential to use methylation patterns of known or unknown genes to identify which patients may benefit from particular chemotherapeutic regimes or biological therapies. Given the potential of opposing effects depending on which genes are methylated, it is vital to examine whether particular methylation events are dominant in conferring resistance. Methods that allow genomewide analysis of methylation patterns may be particularly important for these types of study (Box 1). In a study of latestage ovarian tumours, increased methylation of a subset of $\mathrm{CpG}$ islands significantly correlated with worse clinical outcome, as defined by the time to clinical disease recurrence after chemotherapy (Wei et al, 2002). However in a study of 106 stage III/IV ovarian cancers, methylation of at least one of a group of genes involved in DNA repair/drug detoxification (BRCA1, GSTP1, $M G M T$ ) was associated with improved response to chemotherapy (Teodoridis et al, 2005).

Large-scale analysis of methylation patterns and correlation with response is intrinsically susceptible to the problems of multiple testing. This can be reduced by grouping genes into predefined groups according to a biological hypothesis such as grouping those with similar biological roles or within the same pathway, on the assumption that disruption of any one gene within a pathway or group will disrupt the functioning of that cellular response. This is undoubtedly an oversimplification and the approach will need to be refined as more sophisticated molecular interaction maps and networks are developed (Pommier et al, 2004). An alternative approach will be to use supervised search algorithms that efficiently search array data to identify clusters that associate with clinical outcome (for instance, see Bair and Tibshirani, 2004).

\section{OVERCOMING EPIGENETIC RESISTANCE MECHANISMS}

Epigenetic modifications require active mechanisms of maintenance and so unlike genetic modifications, they are amenable to pharmacological manipulation. 5-Azacytidine and its deoxyribose analogue, 5-aza- $2^{\prime}$-deoxycytidine (decitabine), have been used for many years to inhibit DNA methyltransferases and reverse DNA methylation in tissue culture (Brown and Plumb, 2004). These demethylating agents have been shown to reactivate expression of numerous methylation-silenced genes. Decitabine has clinical activity as a single agent in myelodysplastic syndrome (MDS), CML and AML (Issa et al, 2004). Its activity in solid tumours as a single agent has so far been disappointing. However, it may have a role in sensitising tumours to other anticancer therapies by causing re-expression of genes involved in drug sensitivity (Plumb et al, 2000). In vitro the differentiating effect of decitabine in cultured fibroblasts has a narrow dose window with a loss of action at high doses possibly caused by cytotoxicity as a result of its incorporation into DNA (Taylor and Jones, 1979). It may, therefore, be more appropriate to use demethylating agents at concentrations below the maximally tolerated dose, but still at a level where they are known to cause demethylation and induce gene re-expression. Consistent with this, a low dose schedule appeared to be superior to schedules using higher doses in a study of haematological malignancies (Issa et al, 2004). This has the advantage of reducing the bone marrow toxicity of decitabine and making it easier to combine it with conventional cytotoxics. Histone deacetylase activity is important in the transcriptional repression of methylated sequences (Fischle et al, 2003). The combination of DNA-demethylating agents and HDAC inhibitors causes synergistic re-expression of epigenetically silenced genes (Cameron et al, 1999). It also produces synergistic antitumour effects and increased sensitivity to chemotherapeutic agents in cell line models (Boivin et al, 2002). The potential of this approach is now being assessed in clinical trials (http://www.clinicaltrials.gov/ ct/show/NCT00114257).

Histone deacetylase inhibitors and demethylating agents, such as decitabine, will affect the expression of multiple genes. Given the potential for opposing effects on chemosensitivity when different genes are re-expressed, it could be argued that we need to develop epigenetic therapies that are more gene specific in their mechanism of action. However, if we consider drug resistance to be a polygenic process, then there may be advantages to a multitargeted approach. This implies that some patients may benefit from epigenetic therapies as chemosensitisers, while others will not or may even do worse. Therefore, it will be vital to identify patterns of methylation that reliably predict for response to treatment and whether particular methylation events are dominant in conferring resistance. In order to do this we need robust clinically applicable technology to determine methylation patterns in tumours both at presentation and at relapse. There is also a need for pharmacodynamic markers of response to demethylating agents. Demethylation can be monitored on a whole-genome level or by analysis of individual genes (Lyko and Brown, 2005). It has been shown that genomic DNA methylation levels are decreased in peripheral blood mononuclear cells from xenograft tumourbearing mice treated with 5 -aza- $2^{\prime}$-deoxycytidine (Plumb et al, 2000). This decrease closely coincided with the demethylation of the $h M L H 1$ promoter in the tumours, which indicates that peripheral blood can serve as a surrogate tissue for determining pharmacodynamic characteristics of DNMT inhibitors. However, although demethylation of individual genes such as $p 15$ has been demonstrated in clinical trials (Daskalakis et al, 2002), the 
prognostic value of these methylation changes remains to be established.

\section{CONCLUSIONS}

Aberrant epigenetic regulation, such as DNA methylation of CpG islands, occurs at many genes and in all cancers. CpG island methylation is a potentially important driving force both for tumorogenesis and for drug resistance. The use of demethylating agents and HDAC inhibitors offers the potential to favourably alter the gene expression profile of tumours to cause tumour cell death and increased apoptotic response to established cytotoxic agents. However, we need to identify and evaluate in greater detail the epigenetic characteristics of tumours that predict for lack of response to conventional treatment, so as to identify those patients who may particularly benefit from an epigenetic approach. The polygenic nature of these changes will make this challenging. Towards this objective, genome-wide CpG island methylation of patients' tumours can be examined, as well as detailed characterisation of methylation of individual $\mathrm{CpG}$ islands. Examination of tumour DNA released into body fluids may make the large numbers required for these analyses more feasible. These assays need to be conducted in an appropriate quality assured manner and their utility properly evaluated in prospective, randomised trials. Although the epigenetic therapies now undergoing clinical evaluations show promise, there is a need for further agents, which are more specific for epigenetic targets. This need not equate to more gene specificity, but rather to less nonspecific toxic effects such as the myelosuppression seen with decitabine which may be the result of direct cytotoxic effects of decitabine rather than demethylation. The clinical development of epigenetic therapies will require the development of surrogate pharmacodynamic markers to assess whether these therapies are having their desired pharmacodynamic effect (e.g. global or gene-specific demethylation) and then whether this translates into clinical benefit. Epigenetic pharmacodynamic markers can be used as novel end points in early clinical trials allowing recommended doses to be based on maximal biological effect rather than maximum tolerated dose. Thus, such pharmacodynamic and predictive epigenetic biomarkers, together with targeted drug development, will allow rational and efficient evaluation of novel epigenetic therapies for cancer treatment.

\section{REFERENCES}

Agarwal R, Kaye SB (2003) Ovarian cancer: strategies for overcoming resistance to chemotherapy. Nat Rev Cancer 3: 502-516

Anacleto C, Leopoldino AM, Rossi B, Soares FA, Lopes A, Rocha JC, Caballero O, Camargo AA, Simpson AJ, Pena SD (2005) Colorectal cancer 'methylator phenotype': fact or artifact? Neoplasia 7: 331-335

Bair E, Tibshirani R (2004) Semi-supervised methods to predict patient survival from gene expression data. PLoS Biol 2: E108

Balmain A, Gray J, Ponder B (2003) The genetics and genomics of cancer. Nat Genet 33(Suppl): 238-244

Bhattacharyya NP, Skandalis A, Ganesh A, Groden J, Meuth M (1994) Mutator phenotype in human colorectal carcinoma cell lines. Proc Natl Acad Sci USA 91: 6319-6323

Boivin AJ, Momparler LF, Hurtubise A, Momparler RL (2002) Antineoplastic action of 5 -aza-2'-deoxycytidine and phenylbutyrate on human lung carcinoma cells. Anticancer Drugs 13: $869-874$

Brown R, Plumb JA (2004) Demethylation of DNA by decitabine in cancer chemotherapy. Expert Rev Anticancer Ther 4: 501-510

Cameron EE, Bachman KE, Myohanen S, Herman JG, Baylin SB (1999) Synergy of demethylation and histone deacetylase inhibition in the reexpression of genes silenced in cancer. Nat Genet 21: 103-107

Chang HG, Kim SJ, Chung KW, Noh DY, Kwon Y, Lee ES, Kang HS (2005) Tamoxifen-resistant breast cancers show less frequent methylation of the estrogen receptor beta but not the estrogen receptor alpha gene. J Mol Med 83: $132-139$

Costello JF, Fruhwald MC, Smiraglia DJ, Rush LJ, Robertson GP, Gao X, Wright FA, Feramisco JD, Peltomaki P, Lang JC, Schuller DE, Yu L, Bloomfield CD, Caligiuri MA, Yates A, Nishikawa R, Su Huang H, Petrelli NJ, Zhang X, O'Dorisio MS, Held WA, Cavenee WK, Plass C (2000) Aberrant CpG-island methylation has non-random and tumour-typespecific patterns. Nat Genet 24: $132-138$

Daskalakis M, Nguyen TT, Nguyen C, Guldberg P, Kohler G, Wijermans P, Jones PA, Lubbert M (2002) Demethylation of a hypermethylated P15/ INK4B gene in patients with myelodysplastic syndrome by 5 -aza- $2^{\prime}$ deoxycytidine (decitabine) treatment. Blood 100: 2957-2964

Eads CA, Danenberg KD, Kawakami K, Saltz LB, Blake C, Shibata D, Danenberg PV, Laird PW (2000) MethyLight: a high-throughput assay to measure DNA methylation. Nucleic Acids Res 28: E32

Egger G, Liang G, Aparicio A, Jones PA (2004) Epigenetics in human disease and prospects for epigenetic therapy. Nature 429: 457-463

Esteller M, Corn PG, Baylin SB, Herman JG (2001) A gene hypermethylation profile of human cancer. Cancer Res 61: 3225-3229

Esteller M, Gaidano G, Goodman SN, Zagonel V, Capello D, Botto B, Rossi D, Gloghini A, Vitolo U, Carbone A, Baylin SB, Herman JG (2002) Hypermethylation of the DNA repair gene $\mathrm{O}(6)$-methylguanine DNA methyltransferase and survival of patients with diffuse large B-cell lymphoma. J Natl Cancer Inst 94: 26-32
Esteller M, Garcia-Foncillas J, Andion E, Goodman SN, Hidalgo OF, Vanaclocha V, Baylin SB, Herman JG (2000) Inactivation of the DNArepair gene MGMT and the clinical response of gliomas to alkylating agents. $N$ Engl J Med 343: $1350-1354$

Fischle W, Wang Y, Allis CD (2003) Histone and chromatin cross-talk. Curr Opin Cell Biol 15: 172-183

Fulda S, Kufer MU, Meyer E, van Valen F, Dockhorn-Dworniczak B, Debatin KM (2001) Sensitization for death receptor- or drug-induced apoptosis by re-expression of caspase- 8 through demethylation or gene transfer. Oncogene 20: $5865-5877$

Gerson SL (2004) MGMT: its role in cancer aetiology and cancer therapeutics. Nat Rev Cancer 4: 296-307

Gifford G, Paul J, Vasey PA, Kaye SB, Brown R (2004) The acquisition of hMLH1 methylation in plasma DNA after chemotherapy predicts poor survival for ovarian cancer patients. Clin Cancer Res 10: 4420-4426

Gottesman MM (1993) How cancer cells evade chemotherapy. Cancer Res 53: $747-754$

Grunau C, Clark SJ, Rosenthal A (2001) Bisulfite genomic sequencing: systematic investigation of critical experimental parameters. Nucleic Acids Res 29: E65-5

Hall J, Paul J, Brown R (2004) Critical evaluation of p53 as a prognostic marker in ovarian cancer. Expert Rev Mol Med 2004: 1-20

Hendrich B, Bird A (2000) Mammalian methyltransferases and methylCpG-binding domains: proteins involved in DNA methylation. Curr Top Microbiol Immunol 249: 55-74

Herman JG, Graff JR, Myohanen S, Nelkin BD, Baylin SB (1996) Methylation-specific PCR: a novel PCR assay for methylation status of CpG islands. Proc Natl Acad Sci USA 93: 9821-9826

Huang TH, Perry MR, Laux DE (1999) Methylation profiling of CpG islands in human breast cancer cells. Hum Mol Genet 8: 459-470

Issa JP, Garcia-Manero G, Giles FJ, Mannari R, Thomas D, Faderl S, Bayar E, Lyons J, Rosenfeld CS, Cortes J, Kantarjian HM (2004) Phase 1 study of low-dose prolonged exposure schedules of the hypomethylating agent 5 -aza-2'-deoxycytidine (decitabine) in hematopoietic malignancies. Blood 103: $1635-1640$

Johnson PJ, Lo YM (2002) Plasma nucleic acids in the diagnosis and management of malignant disease. Clin Chem 48: 1186-1193

Lachner M, O'Sullivan RJ, Jenuwein T (2003) An epigenetic road map for histone lysine methylation. J Cell Sci 116: 2117-2124

Loeb LA (1994) Microsatellite instability: marker of a mutator phenotype in cancer. Cancer Res. 54: 5059-5063

Lowe SW, Ruley HE, Jacks T, Housman DE (1993) p53-dependent apoptosis modulates the cytotoxicity of anticancer agents. Cell 74: 957-967

Lyko F, Brown R (2005) DNA methyltransferase inhibitors and the development of epigenetic cancer therapies. J Natl Cancer Inst 97: $1498-1506$ 
Papouli E, Cejka P, Jiricny J (2004) Dependence of the cytotoxicity of DNAdamaging agents on the mismatch repair status of human cells. Cancer Res 64: $3391-3394$

Paz MF, Avila S, Fraga MF, Pollan M, Capella G, Peinado MA, SanchezCespedes M, Herman JG, Esteller M (2002) Germ-line variants in methylgroup metabolism genes and susceptibility to DNA methylation in normal tissues and human primary tumors. Cancer Res 62: 4519-4524

Paz MF, Yaya-Tur R, Rojas-Marcos I, Reynes G, Pollan M, Aguirre-Cruz L, Garcia-Lopez JL, Piquer J, Safont MJ, Balana C, Sanchez-Cespedes M, Garcia-Villanueva M, Arribas L, Esteller M (2004) CpG island hypermethylation of the DNA repair enzyme methyltransferase predicts response to temozolomide in primary gliomas. Clin Cancer Res 10: $4933-4938$

Plumb JA, Strathdee G, Sludden J, Kaye SB, Brown R (2000) Reversal of drug resistance in human tumor xenografts by $2^{\prime}$-deoxy-5-azacytidineinduced demethylation of the hMLH1 gene promoter. Cancer Res 60: 6039-6044

Pommier Y, Sordet O, Antony S, Hayward RL, Kohn KW (2004) Apoptosis defects and chemotherapy resistance: molecular interaction maps and networks. Oncogene 23: 2934-2949

Richardson BC (2002) Role of DNA methylation in the regulation of cell function: autoimmunity, aging and cancer. J Nutr 132: 2401S-2405S

Soengas MS, Capodieci P, Polsky D, Mora J, Esteller M, Opitz-Araya X, McCombie R, Herman JG, Gerald WL, Lazebnik YA, Cordon-Cardo C, Lowe SW (2001) Inactivation of the apoptosis effector Apaf-1 in malignant melanoma. Nature 409: 207-211

Strathdee G, MacKean MJ, Illand M, Brown R (1999) A role for methylation of the hMLH1 promoter in loss of hMLH1 expression and drug resistance in ovarian cancer. Oncogene 18: 2335-2341

Strathdee G, Vass JK, Oien KA, Siddiqui N, Curto-Garcia J, Brown R (2005) Demethylation of the MCJ gene in stage III/IV epithelial ovarian cancer and response to chemotherapy. Gynecol Oncol 97: 898-903

Taniguchi T, Tischkowitz M, Ameziane N, Hodgson SV, Mathew CG, Joenje H, Mok SC, D’Andrea AD (2003) Disruption of the Fanconi anemiaBRCA pathway in cisplatin-sensitive ovarian tumors. Nat Med 9: $568-574$

Taylor SM, Jones PA (1979) Multiple new phenotypes induced in 10T1/2 and 3T3 cells treated with 5-azacytidine. Cell 17: 771-779

Teodoridis JM, Hall J, Marsh S, Kannall HD, Smyth C, Curto J, Siddiqui N, Gabra H, McLeod HL, Strathdee G, Brown R (2005) CpG island methylation of DNA damage response genes in advanced ovarian cancer. Cancer Res 65: $8961-8967$

Teodoridis JM, Strathdee G, Brown R (2004) Epigenetic silencing mediated by $\mathrm{CpG}$ island methylation: potential as a therapeutic target and as a biomarker. Drug Resist Update 7: 267-278

Tra J, Kondo T, Lu Q, Kuick R, Hanash S, Richardson B (2002) Infrequent occurrence of age-dependent changes in $\mathrm{CpG}$ island methylation as detected by restriction landmark genome scanning. Mech Ageing Dev 123: $1487-1503$

Toyota M, Ahuja N, Ohe-Toyota M, Herman JG, Baylin SB, Issa JP (1999) $\mathrm{CpG}$ island methylator phenotype in colorectal cancer. Proc Natl Acad Sci USA 96: $8681-8686$

Ushijima T, Morimura K, Hosoya Y, Okonogi H, Tatematsu M, Sugimura T, Nagao M (1997) Establishment of methylation-sensitive-representational difference analysis and isolation of hypo- and hypermethylated genomic fragments in mouse liver tumors. Proc Natl Acad Sci USA 94: $2284-2289$

Valk-Lingbeek ME, Bruggeman SW, van Lohuizen M (2004) Stem cells cancer; the polycomb connection. Cell 118: $409-418$

Warnecke PM, Stirzaker C, Song J, Grunau C, Melki JR, Clark SJ (2002) Identification and resolution of artifacts in bisulfite sequencing. Methods 27: $101-107$

Wei SH, Brown R, Huang TH (2003) Aberrant DNA methylation in ovarian cancer: is there an epigenetic predisposition to drug response? Ann N Y Acad Sci 983: $243-250$

Wei SH, Chen CM, Strathdee G, Harnsomburana J, Shyu CR, Rahmatpanah F, Shi H, Ng SW, Yan PS, Nephew KP, Brown R, Huang TH (2002) Methylation microarray analysis of late-stage ovarian carcinomas distinguishes progression-free survival in patients and identifies candidate epigenetic markers. Clin Cancer Res 8: 2246-2252

Xiong Z, Laird PW (1997) COBRA: a sensitive and quantitative DNA methylation assay. Nucleic Acids Res 25: 2532-2534

Yamashita K, Dai T, Dai Y, Yamamoto F, Perucho M (2003) Genetics supersedes epigenetics in colon cancer phenotype. Cancer Cell 4: $121-131$

Zhang L, Conejo-Garcia JR, Katsaros D, Gimotty PA, Massobrio M, Regnani G, Makrigiannakis A, Gray H, Schlienger K, Liebman MN, Rubin SC, Coukos G (2003) Intratumoral $\mathrm{T}$ cells, recurrence, and survival in epithelial ovarian cancer. $N$ Engl J Med 348: 203-213 